\section{(A) Check for updates}

Cite this: Polym. Chem., 2021, 12 2712

\title{
Synthesis, characterization and self-assembly of linear and miktoarm star copolymers of exclusively immiscible polydienes $\uparrow$
}

\author{
Konstantinos Ntetsikas, (DD ${ }^{a, b}$ Dimitrios Moschovas, ${ }^{a, c}$ George Zapsas, (D) a,b \\ loannis Moutsios, (iD a Konstantina Tsitoni, ${ }^{a}$ Gkreti-Maria Manesi, (iD a \\ Azat F. Nabiullin, ${ }^{c, d}$ Nikos Hadjichristidis, (iD b Dimitri A. Ivanov (iD c,d,e and \\ Apostolos Avgeropoulos iD *a,c
}

\begin{abstract}
Linear and non-linear copolymers of the PB-b-PI sequence [PB: polybutadiene of high 1,4-microstructure ( 92\%) and PI: polyisoprene of high 3,4-microstructure ( 55-60\%)] and their corresponding miktoarm star copolymers of the $\mathrm{PB}\left(\mathrm{Pl}_{3,4}\right)_{2}$ and $\mathrm{PB}\left(\mathrm{Pl}_{3,4}\right)_{3}$ type were synthesized by combining anionic polymerization and selective chlorosilane chemistry. Molecular characteristics, thermal properties and structure/ properties relationship are reported for the specific copolymers and especially the self-assembly is of major importance and interest due to the nature of the blocks. The identical electron densities between the two polydienes led to impossible morphological characterization through small angle $\mathrm{X}$-ray scattering (SAXS) and only transmission electron microscopy results verify the adopted morphology for each copolymer, justifying the assumption that the segment-segment interaction parameter between the two polydienes of high 1,4-microstructure ( 92\%) for the PB and $55-60 \%$ 3,4-microstructure for the PI is well above zero. The consistency of the bulk morphology results of this study compared with those of the extensively studied system of the PS(PI) $n=1,2,3$ type (PS: polystyrene), were unexpectedly coherent. High chain flexibility provided by the two polydiene segments, leads to promising properties unattainable from corresponding thermoplastic triblock copolymers of these polydienes with PS (PS- $b-$ PI- $b-$ PS, PS- $b-$ PB$b$-PS), especially for rheological studies.
\end{abstract}

Received 25th February 2021, Accepted 12th April 2021

DOI: $10.1039 /$ d1py00258a

rsc.li/polymers

\section{Introduction}

Diblock copolymers constitute a highly investigated topic, due to their exceptional properties, rendering them suitable for various applications..$^{1-4}$ Their unique potential is attributed to their self-assembly in several nanostructures, which is determined by the value $\chi N(\chi$ : Flory-Huggins interaction parameter and $N$ : degree of polymerization $)^{5}$ and the volume fraction of at least one of the blocks $\left(\varphi_{\mathrm{A}}\right.$ since $\left.\varphi_{\mathrm{B}}=1-\varphi_{\mathrm{A}}\right)$. Recently, linear

\footnotetext{
${ }^{a}$ Department of Materials Science Engineering, University of Ioannina, University Campus-Dourouti, 45110 Ioannina, Greece.E-mail: aavger@uoi.gr

${ }^{b}$ Physical Sciences and Engineering Division, KAUST Catalysis Center, Polymer Synthesis Laboratory, King Abdullah University of Science and Technology (KAUST), Thuwal 23955, Saudi Arabia

${ }^{c}$ Faculty of Chemistry, Lomonosov Moscow State University (MSU), GSP-1, 1-3 Leninskiye Gory, 119991 Moscow, Russia

${ }^{d}$ Institute of Problems of Chemical Physics, Russian Academy of Sciences, Chernogolovka, 142432 Moscow, Russia

${ }^{e}$ Institut de Sciences des Matériaux de Mulhouse - IS2M, CNRS UMR7361, 15 Jean Starcky, Mulhouse 68057, France

$\dagger$ Electronic supplementary information (ESI) available. See DOI: 10.1039/ d1py00258a
}

diblock copolymers, exhibiting high segment incompatibility, have been thoroughly studied due to their prospective use in nanotechnology, ${ }^{6,7}$ optoelectronics ${ }^{8}$ etc. Advances on anionic and controlled/living radical polymerization techniques provide the opportunity of synthesizing complex architecture copolymers and terpolymers, ${ }^{9-13}$ leading to enhanced properties of these materials when compared to their linear analogues. ${ }^{14}$ Microphase separation of miktoarm star copolymers particularly of the $\mathrm{AB}_{n}$ type (where $n=2,3$, etc.) results in differentiations on the expected self-assembly topologies, and unique morphologies are obtained, due to the restrictions imposed by the single junction point on which all chains are connected. ${ }^{12,15,16}$

The morphologies adopted from miktoarm star copolymers depend on the total degree of polymerization $(N)$, the volume fraction $(\varphi)$, the A/B segment-segment Flory-Huggins interaction parameter $(\chi)$, the molecular architecture and the elasticity parameter $(\varepsilon)$, which describes the effect of both chain architecture and elastic asymmetry in the strong-segregation regime. ${ }^{17-19}$ Although, synthesis and microphase separation of complex architecture systems, containing a number of different dissimilar segments, have been analytically investigated in the literature, ${ }^{9}$ only a few cases involving elastomeric 
blocks and specifically polyisoprene (PI) and polybutadiene (PB) have been reported. ${ }^{20-22}$ The possible segment microstructures for PB are: 1,4-cis, 1,4-trans and 1,2-, while for PI are: 1,4-cis, 1,4-trans, 3,4- and 1,2. When the 3,4-microstructure of PI is significantly increased $(\sim 55-60 \%)$, then the 1,2-microstructure is increased as well (15-25\%) leading to a small percentage for the 1,4 linear segments. In general, the possible combinations of microstructures for PB and PI in a diblock copolymer result in the following sequences: $\mathrm{PB}_{1,4}-b-\mathrm{PI}_{1,4}$, $\mathrm{PB}_{1,2}-b-\mathrm{PI}_{1,4}, \mathrm{~PB}_{1,2}-b-\mathrm{PI}_{3,4}$ and $\mathrm{PB}_{1,4}-b-\mathrm{PI}_{3,4} \cdot{ }^{23,24}$

For block copolymers, the type and relative amount of the specific stereoisomers should always be taken into consideration, since local structure influences the segment-segment interaction parameter. The aforementioned combinations of polydiene chains, namely $\mathrm{PB}_{1,4}-b-\mathrm{PI}_{1,4}, \mathrm{~PB}_{1,2}-b-\mathrm{PI}_{1,4}$ and $\mathrm{PB}_{1,2^{-}}$ $b$ - $\mathrm{PI}_{3,4}$, have been explored, and the interaction parameters were found sufficiently low, concluding to mixed homogeneous phases, either for copolymers or even blends of the polydiene pairs in typical molecular weights. The $\chi$ interaction parameter of diblock copolymers of predominantly 1,4-PB and predominantly cis-1,4 PI with either or both of the corresponding linear homopolymers has been determined. ${ }^{23}$ Cohen and Wilfong ${ }^{24}$ calculated $\chi$ for different diene pairs, obtaining room temperature values of 0.081 and 0.048 for $1,4-\mathrm{PI} / 1,4-\mathrm{PB}$ and 1,4-PI/1,2-PB sequences, respectively.

The synthesis and morphological characterization of linear terpolymers containing PS, PB and PI have been reported for the first time by Neumann et al. ${ }^{25,26}$ The results of this study showed that the specific triblocks behaved mainly as twophase systems, consisting of a mixed 1,4-PI/1,2-PB phase segregated by the polystyrene (PS) domains. Avgeropoulos et al. ${ }^{27}$ reported for the first time anionically synthesized and morphologically characterized ABC triblock terpolymers with two polydiene blocks and one PS, where the difference lies in the 3,4microstructure content of the PI ( 55\% 3,4-content), whereas the PB block was of high 1,4-microstructure ( 92\%). A threecomponent microphase separated system was adopted, leading to the conclusion that the $1,4-\mathrm{PB} / 3,4-\mathrm{PI}$ polydiene combination has a comparatively higher interaction parameter $\chi$ than the other three possible combinations (1,4-PI/1,4-PB, 1,4$\mathrm{PI} / 1,2-\mathrm{PB}$ and 3,4-PI/1,2-PB). Furthermore, altering the block sequence from PB- $b$-PS- $b$-PI to PS- $b$-PB- $b$-PI, but keeping the volume fraction ratio constant between the segments, resulted in identical morphologies (three-phase four-layer lamellae), leading to the conclusion that the adopted topologies were equilibrium structures [since the block sequence did not alter the morphology as already reported in PS- $b-\mathrm{PI}-b-\mathrm{P} 2 \mathrm{VP}^{28} v s$. PI$b$-PS- $b$-P2 $2 \mathrm{VP}^{29}$ respectively, where $\mathrm{P} 2 \mathrm{VP}$ corresponds to poly(2vinylpyridine)].

Additionally, Avgeropoulos' group ${ }^{22}$ showed the coexistence of core-shell double gyroid and three-phase four-layer lamellar morphologies for similar PS- $b$-PB- $b$-PI triblock terpolymers, as verified by transmission electron microscopy. SAXS results led to the conclusion that the 3-phase 4-layer lamellae are most evident, despite the relatively long-range order and the observations of both structures by bright-field TEM images. Diblock copolymers of the $\mathrm{PB}_{1,4}-b-\mathrm{PI}_{3,4}$ type were also synthesized, in order to verify the microphase separation and immiscibility of these two polydienes with specific geometric isomerisms. ${ }^{22}$

Further investigations were reported on more complex architecture materials, such as second-generation dendritic copolymers consisted of polydienic segments exclusively and terpolymers comprised of two polydiene blocks and one relatively rigid block (PS). These studies led to self-assembled twophase and three-phase topologies, despite the complexity of these systems, indicating that the interaction parameter, especially between the two polydienes is well above zero. ${ }^{30-32}$

Finally, an alternating gyroid morphology has been for the first time reported in the literature for an $\mathrm{ABC}$ miktoarm star terpolymer consisting of PS, PB and PI, where the two polydienes exhibited respective microstructures ( $92 \%$ 1,4-microstructure for PB and $\sim 55-60 \%$ 3,4-microstructure for PI). ${ }^{33}$

Apart from their self-assembly capability, polymers consisted of at least one elastomeric unit exhibit interest in the field of rheology, due to their fatigue resistance and viscoelastic properties, rendering them appealing materials as adhesives for industrial applications. ${ }^{34-40}$

In this study, we report the synthesis of four (4) linear diblock copolymers of the $\mathrm{PB}_{1,4}-b-\mathrm{PI}_{3,4}$ sequence, four (4) asymmetric miktoarm star copolymers of the $\mathrm{PB}_{1,4}\left(\mathrm{PI}_{3,4}\right)_{2}$, and four (4) of the $\mathrm{PB}_{1,4}\left(\mathrm{PI}_{3,4}\right)_{3}$ type. The synthesis procedure and properties of such non-linear copolymers have not been reported in the literature yet.

The molecular characterization of all samples was performed through size exclusion chromatography (SEC), to confirm the dispersity $(\nexists)$, and membrane osmometry (MO) to calculate the number average molecular weight, $\left(\bar{M}_{\mathrm{n}}\right)$ values. Moreover, proton nuclear magnetic resonance spectroscopy $\left({ }^{1} \mathrm{H}-\mathrm{NMR}\right)$ was employed to verify the characteristic ratios of stereochemical microstructures for the polydienes as well as to identify the composition of each segment. Thermal analysis via differential scanning calorimetry (DSC) was also performed to examine the glass transition temperatures $\left(T_{\mathrm{g}}\right)$ of the two blocks, their potential microphase separation when studied in bulk, and the dependence of the architecture on the $T_{\mathrm{g}}$ of each system.

Morphological characterization was carried out exclusively through bright-field transmission electron microscopy (TEM), to verify the microphase separation and provide significant information concerning the dependence of the complex architecture on the adopted morphology. It should be mentioned that the total average molecular weight of the PB block, as well as the volume fraction, remained almost identical in both linear and non-linear copolymers in order to compare their structure/properties relationship and verify the influence of non-linear architecture in the self-assembly of such systems.

\section{Experimental}

\section{Materials}

1,3-Butadiene (99\%), trichloromethylsilane $\left(\mathrm{CH}_{3} \mathrm{SiCl}_{3}\right)$ (99\%), tetrachlorosilane $\left(\mathrm{SiCl}_{4}\right)(99 \%)$, and calcium hydride $\left(\mathrm{CaH}_{2}\right)$ 
(95\%) were purchased from Sigma-Aldrich, isoprene (98\%), sec-butyllithium, $n$-butyllithium and styrene (99\%) from Acros Organics, benzene (99.7\%) from Chem-Lab, tetrahydrofuran (THF) (99.9\%) from Carlo Erba, toluene (99.7\%) from Honeywell and methanol (99\%) from Fluka. The purification methods of all reagents involved in the polymerizations were carried out based on the requirements of anionic polymerization and are well elaborated elsewhere. ${ }^{41,42}$

\section{Instrumentation}

Size exclusion chromatography (SEC) measurements were carried out using a SpectraSystem P1000 equipped with an isocratic pump, column oven (LabAlliance) heated at $30^{\circ} \mathrm{C}$, three columns in series (PLgel $5 \mathrm{~mm}$ Mixed-C, $3007.5 \mathrm{~mm}$ ), refractive index (RI, Shodex RI-101) and ultraviolet absorbance (UV, SpectraSystem UV1000) detectors. Tetrahydrofuran (THF) was

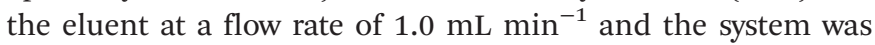
calibrated with eight PS standards $\left(M_{\mathrm{p}}: 4300\right.$ to $3000000 \mathrm{~g}$ $\left.\mathrm{mol}^{-1}\right)$.

Membrane osmometry (MO) was adopted to determine the number average molecular weight by using a Gonotec Osmomat 090 in $35^{\circ} \mathrm{C}$ using toluene as solvent.

Proton nuclear magnetic resonance $\left({ }^{1} \mathrm{H}-\mathrm{NMR}\right)$ spectroscopy was carried out in $\mathrm{CDCl}_{3}$ at $25^{\circ} \mathrm{C}$ using Bruker AVANCE II spectrometers operating at 250 and $400 \mathrm{MHz}$ and data were processed using UXNMR (Bruker) software.

Differential scanning calorimetry (DSC) measurements were performed on a Q20 TA instrument. The heating ramp was $5{ }^{\circ} \mathrm{C} \min ^{-1}$ and the temperature range from $-120{ }^{\circ} \mathrm{C}$ to $40{ }^{\circ} \mathrm{C}$. A small amount of $5 \mathrm{mg}$ was used from each sample. Two heating and one cooling cycles were performed and the results of the second heating were reported and analysed using Advantage v5.4.0 (TA instruments) software.

Transmission electron microscopy (TEM) experiments were performed in a JEOL 2100 TEM using $200 \mathrm{keV}$ as the acceleration voltage. Cryo-ultramicrotoming of the as-cast films was performed in a Leica EM UC7 ultramicrotome, in order to obtain very thin sections $(\sim 40 \mathrm{~nm})$ at $-100{ }^{\circ} \mathrm{C}$ (below the lowest $T_{\mathrm{g}}$ of both $\mathrm{PB}_{1,4}$ and $\mathrm{PI}_{3,4}$, being approximately $-90{ }^{\circ} \mathrm{C}$ and $-10{ }^{\circ} \mathrm{C}$ respectively) and the sections were picked up on 600 mesh copper grids. Since both blocks are exclusively consisting of carbon and hydrogen atoms, selective staining with vapors of $\mathrm{OsO}_{4} 4 \%$ aqueous solution was employed for $\sim 60$ minutes (based on the aging of the stainer solution).

\section{Synthesis of linear and non-linear diblock copolymers}

A thorough description of the synthetic protocol for the linear diblock copolymers of the $\mathrm{PB}_{1,4}-b-\mathrm{PI}_{3,4}$ type has already been mentioned in the literature by Zapsas et al. ${ }^{22}$ However, the novel synthesis of the miktoarm star copolymers consisting of only polydiene segments is documented for the first time. Specifically, four (4) miktoarm star copolymers of the $\mathrm{PB}_{1,4}\left(\mathrm{PI}_{3,4}\right)_{2}$ and four (4) of the $\mathrm{PB}_{1,4}\left(\mathrm{PI}_{3,4}\right)_{3}$ type were synthesized following the justified synthetic protocols of anionic polymerization and chlorosilane chemistry, under high vacuum conditions. The anionic polymerization and linking reactions were carried out in evacuated, $n$-BuLi washed custom-made glass reactors, at room temperature. Reagents were added via break-seals and aliquots for characterization were taken by heat-sealing of constrictions at all steps.

The analytical adopted synthetic procedure for the preparation of the miktoarm star copolymers of the $\mathrm{PB}_{1,4}\left(\mathrm{PI}_{3,4}\right)_{2,3}$ type belonging to set no. 1 is described: following the purification of 1,3-butadiene ( $15 \mathrm{~g}, 0.27 \mathrm{~mol}$ ), the monomer was distilled in the apparatus, containing purified benzene $(500 \mathrm{~mL})$ and subsequently, sec-BuLi $(0.34 \mathrm{mmol})$ was introduced to the solution leading to initiation of the 1,3-butadiene polymerization, which was completed after 24 hours at room temperature (Fig. 1a). A small aliquot was taken for the molecular characterization of the PB segment via SEC, MO and ${ }^{1} \mathrm{H}-\mathrm{NMR}$. The living $\mathrm{PB}_{1,4}$ chains reacted instantly and under continuous stirring with excess (at least 500-fold excess $\sim 0.17 \mathrm{~mol}$ ) of linking chlorosilane reagents (either $\mathrm{CH}_{3} \mathrm{SiCl}_{3}$ or $\mathrm{SiCl}_{4}$ ), to exclusively substitute just one chlorine atom as shown in Fig. $1 \mathrm{~b}$ for the case of $\mathrm{CH}_{3} \mathrm{SiCl}_{3}$ and in Fig. 1c for $\mathrm{SiCl}_{4}$. After the complete removal of the excess of the linking reagent on the high vacuum line, a substantial amount of purified benzene was distilled in the apparatus to re-dilute the $\mathrm{PB}_{1,4}$-Si$\mathrm{Cl}_{2}$ or the $\mathrm{PB}_{1,4}-\mathrm{Si}-\mathrm{Cl}_{3}$ intermediate product. To a separate glass apparatus, an appropriate amount of isoprene $(21.5 \mathrm{~g}$, $0.31 \mathrm{~mol})$ and $s e c$-BuLi $(0.6 \mathrm{mmol})$ in a mixture of non-polar solvent (benzene, $500 \mathrm{~mL}$ ) and polar solvent (THF, $1 \mathrm{~mL}$ ) were introduced under high vacuum and were left to react at room

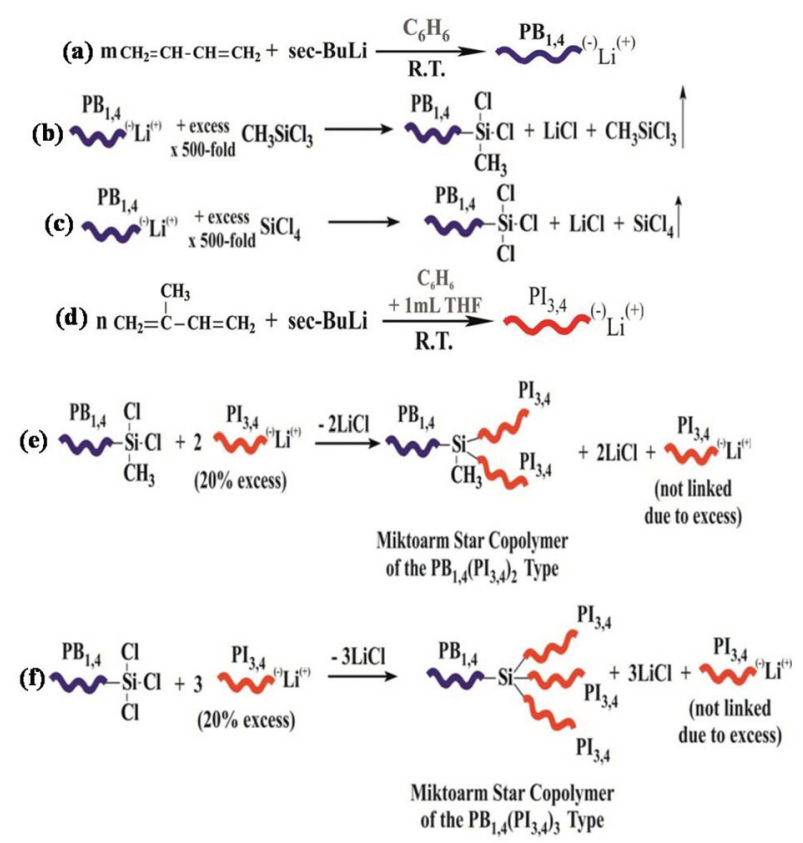

Fig. 1 Schematic illustration of the synthesis of the $\mathrm{PB}_{1,4}\left(\mathrm{PI}_{3,4}\right)_{2}$ and $\mathrm{PB}_{1,4}\left(\mathrm{PI}_{3,4}\right)_{3}$ miktoarm star copolymers. (a) Synthetic route for the living $\mathrm{PB}$ precursor, $\mathrm{PB}_{1,4}{ }^{(-)} \mathrm{Li}^{(+)}$, (b) coupling reaction between trichloromethylsilane and $\mathrm{PB}_{1,4}{ }^{(-)} \mathrm{Li}^{(+)}$, (c) coupling reaction between silicon tetrachloride and $\mathrm{PB}_{1,4}{ }^{(-)} \mathrm{Li}^{(+)}$, (d) synthesis reaction of the living $\mathrm{PI}$ chains, $\mathrm{PI}_{3,4}{ }^{(-)} \mathrm{Li}^{(+)}$, (e) linking reaction for the synthesis of miktoarm star copolymer of the $\mathrm{PB}_{1,4}\left(\mathrm{PI}_{3,4}\right)_{2}$ type and $(\mathrm{f})$ corresponding linking reaction for miktoarm star copolymer of the $\mathrm{PB}_{1,4}\left(\mathrm{PI}_{3,4}\right)_{3}$ type. 
temperature for 24 hours (Fig. 1d) to synthesize the living $\mathrm{PI}_{3,4}{ }^{(-)} \mathrm{Li}^{(+)}$exhibiting the required 3,4-microstructure $(\sim 55-60 \%)$. In the case of $\mathrm{PB}_{1,4}\left(\mathrm{PI}_{3,4}\right)_{3}$ different amount of isoprene was employed, specifically $14.5 \mathrm{~g}$ of monomer $(0.21 \mathrm{~mol})$ were reacted with $0.6 \mathrm{mmol} s e c$-BuLi. An excess of the $\mathrm{PI}_{3,4}{ }^{(-)} \mathrm{Li}^{(+)}(\sim 20 \%$ excess compared to the Si-Cl living ends of the $\mathrm{PB}_{1,4}-\mathrm{Si}_{-} \mathrm{Cl}_{2}$ or $\mathrm{PB}_{1,4}-\mathrm{Si}_{-}-\mathrm{Cl}_{3}$ ) was introduced in the polymerization reactor substituting all remaining chlorine atoms evident in the $\mathrm{PB}_{1,4}-\mathrm{Si}-\mathrm{Cl}_{2}$ or the $\mathrm{PB}_{1,4}-\mathrm{Si}_{-}-\mathrm{Cl}_{3}$ intermediate products, leading eventually to the desired $\mathrm{PB}_{1,4}\left(\mathrm{PI}_{3,4}\right)_{2}$ and $\mathrm{PB}_{1,4}\left(\mathrm{PI}_{3,4}\right)_{3}$ miktoarm star copolymers respectively (Fig. 1e and $\mathrm{f})$.

\section{Results and discussion}

\section{Molecular characterization results of linear diblock and non- linear miktoarm star copolymers}

The molecular characterization results for all samples [linear diblock copolymers of the $\mathrm{PB}_{1,4}-b$ - $\mathrm{PI}_{3,4}$ type and miktoarm star copolymers of the $\mathrm{PB}_{1,4}\left(\mathrm{PI}_{3,4}\right)_{2}$ and $\mathrm{PB}_{1,4}\left(\mathrm{PI}_{3,4}\right)_{3}$ type respectively] are presented in Table 1 indicating increased compositional and molecular homogeneity. The mass fraction, $f$, was calculated via ${ }^{1} \mathrm{H}-\mathrm{NMR}$ measurements. For comparison reasons, the samples were divided into 4 different sets, where each set includes one linear diblock copolymer with similar molecular characteristics to the corresponding $\mathrm{PB}_{1,4}\left(\mathrm{PI}_{3,4}\right)_{2}$ and $\mathrm{PB}_{1,4}\left(\mathrm{PI}_{3,4}\right)_{3}$ miktoarm stars. The glass transition temperatures for all final materials were determined via DSC. The dispersity indices were retrieved by SEC measurements and the number average molecular weights $\left(\bar{M}_{\mathrm{n}}\right)$ for the individual blocks and the final copolymers were calculated by MO.

All diblock copolymers were synthesized through anionic polymerization by using the sequential monomer addition method and high vacuum techniques, while for the non-linear copolymers, selective chlorosilane chemistry was employed. High 3,4-microstructure was adopted for all PI blocks by using a small amount $(\sim 1 \mathrm{~mL})$ of a polar additive (THF). The total number average molecular weight of the final copolymers, in all cases, was kept constant at approximately $100 \mathrm{~kg} \mathrm{~mol}^{-1}$, to compare the molecular and morphological characteristics of the linear diblock copolymers with the respective miktoarm stars. Furthermore, such diblock copolymers with the specific segments and microstructure content have been sparsely synthesized and studied in bulk up to date. ${ }^{22}$ It is important to mention that these types of miktoarm star copolymers were synthesized for the first time and have never been reported in the literature. In all cases, $\mathrm{PB}$ was the first block $\left(\mathrm{PB}_{1,4}-b-\mathrm{PI}_{3,4}\right.$ sequence) since it is not possible to synthesize well-defined $\mathrm{PI}_{3,4}-b$ - $\mathrm{PB}_{1,4}$ taking into account that the presence of THF from the beginning of the polymerization (when PI is the first block) would increase the 1,2-microstructure of $\mathrm{PB}$ and no microphase separation would be observed.

As already reported in the literature, microphase separation between the two blocks is evident only when the 3,4-microstructure content of the PI is high $(\sim 55-60 \%)$, and that of the PB block shows high 1,4-microstructure ( 92\%). ${ }^{22-24,31}$

The monomodal molecular weight distributions of the four linear diblock copolymers of the $\mathrm{PB}_{1,4}-b-\mathrm{PI}_{3,4}$ type, indicating high molecular and compositional homogeneity, as well as the absence of any undesired by-products during the synthetic procedure, are observed in Fig. 2a. In the ESI, (Fig. S1 and S2 $\dagger$ ), two representative chromatographs of the linear samples $\left(\mathrm{PB}_{1,4}-b-\mathrm{PI}_{3,4}-3\right.$ and $\left.\mathrm{PB}_{1,4}-b-\mathrm{PI}_{3,4}-4\right)$ are depicted separately along with the initial $\mathrm{PB}$ precursors.

For all miktoarm star copolymer samples, the total number average molecular weight varied between $88-109 \mathrm{~kg} \mathrm{~mol}{ }^{-1}$, in agreement with the corresponding values mentioned already for the linear diblock copolymers. Additionally, the number average molecular weight of the $\mathrm{PB}$ arms for each set is kept constant. Accordingly, narrow dispersity indices for all miktoarm star copolymers are illustrated in Fig. $2 \mathrm{~b}$ and c, respectively. In the ESI, (Fig. S3 and S4 $\dagger$ ) the SEC chromatographs of the $\mathrm{PB}$ and PI blocks, the unfractionated and the final fractionated miktoarm star copolymers of the $\mathrm{PB}_{1,4}\left(\mathrm{PI}_{3,4}\right)_{2}-\mathrm{S}_{7}$ and $\mathrm{PB}_{1,4}\left(\mathrm{PI}_{3,4}\right)_{3}-\mathrm{S}_{8}$ are shown separately. The solvent/non-solvent fractionation technique was used to remove the undesired products, which were formed during the linking reactions of the

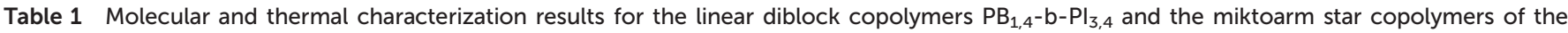
$\mathrm{PB}_{1,4}\left(\mathrm{PI}_{3,4}\right)_{2}$ and $\mathrm{PB}_{1,4}\left(\mathrm{PI}_{3,4}\right)_{3}$ types

\begin{tabular}{|c|c|c|c|c|c|c|c|c|c|}
\hline Sample sets & Samples & $\left(\bar{M}_{\mathrm{n}}\right)_{\mathrm{PB}}{ }^{a}\left(\mathrm{~kg} \mathrm{\textrm {mol } ^ { - 1 }}\right)$ & $\left(\bar{M}_{\mathrm{n}}\right)_{\mathrm{PI}}^{a}\left(\mathrm{~kg} \mathrm{~mol}^{-1}\right)$ & $\left(\bar{M}_{\mathrm{n}}\right)_{\text {total }}{ }^{a}\left(\mathrm{~kg} \mathrm{~mol}^{-1}\right)$ & $\bigoplus_{\text {total }}^{b}$ & $f_{\mathrm{PB}}^{c}$ & $\varphi_{\mathrm{PB}}^{d}$ & $\left(T_{\mathrm{g}}\right)_{\mathrm{PB}}^{e}\left({ }^{\circ} \mathrm{C}\right)$ & $\left(T_{\mathrm{g}}\right)_{\mathrm{PI}}{ }^{e}\left({ }^{\circ} \mathrm{C}\right)$ \\
\hline \multirow[t]{3}{*}{ Set no. 1} & $\mathrm{~PB}_{1,4}-b-\mathrm{PI}_{3.4^{-1}}$ & 38.2 & 55.4 & 93.6 & 1.06 & 0.42 & 0.42 & -91 & -8 \\
\hline & $\mathrm{PB}_{1,4}\left(\mathrm{PI}_{3.4}\right)_{2}-\mathrm{S}_{1}$ & 43.1 & 35.3 & 107.3 & 1.08 & 0.41 & 0.41 & -91 & -7 \\
\hline & $\mathrm{PB}_{1,4}\left(\mathrm{PI}_{3.4}\right)_{3}-\mathrm{S}_{2}$ & 43.1 & 23.8 & 109.4 & 1.07 & 0.40 & 0.40 & -91 & -5 \\
\hline \multirow[t]{3}{*}{ Set no. 2} & $\mathrm{~PB}_{1,4}-b-\mathrm{PI}_{3.4}-2$ & 65.1 & 27.3 & 92.4 & 1.05 & 0.71 & 0.71 & -91 & -7 \\
\hline & $\mathrm{PB}_{1,4}\left(\mathrm{PI}_{3.4}\right)_{2}-\mathrm{S}_{3}$ & 61.5 & 16.7 & 92.4 & 1.06 & 0.67 & 0.67 & -91 & -4 \\
\hline & $\mathrm{PB}_{1,4}\left(\mathrm{PI}_{3.4}\right)_{3}-\mathrm{S}_{4}$ & 61.5 & 9.8 & 88.2 & 1.08 & 0.69 & 0.69 & -91 & -6 \\
\hline \multirow[t]{3}{*}{ Set no. 3} & $\mathrm{~PB}_{1,4}-b-\mathrm{PI}_{3.4}-3$ & 58.3 & 40.2 & 98.5 & 1.06 & 0.59 & 0.59 & -91 & -11 \\
\hline & $\mathrm{PB}_{1,4}\left(\mathrm{PI}_{3.4}\right)_{2}-\mathrm{S}_{5}$ & 55.2 & 22.8 & 98.1 & 1.06 & 0.57 & 0.57 & -91 & -11 \\
\hline & $\mathrm{PB}_{1,4}\left(\mathrm{PI}_{3.4}\right)_{3}-\mathrm{S}_{6}$ & 55.2 & 15.6 & 100.3 & 1.07 & 0.56 & 0.56 & -91 & -10 \\
\hline \multirow[t]{3}{*}{ Set no. 4} & $\mathrm{~PB}_{1,4}-b-\mathrm{PI}_{3.4}-4$ & 35.5 & 72.4 & 107.9 & 1.07 & 0.32 & 0.32 & -92 & -18 \\
\hline & $\mathrm{PB}_{1,4}\left(\mathrm{PI}_{3.4}\right)_{2}-\mathrm{S}_{7}$ & 27.5 & 34.5 & 94.5 & 1.05 & 0.30 & 0.30 & -92 & -17 \\
\hline & $\mathrm{PB}_{1,4}\left(\mathrm{PI}_{3.4}\right)_{3}-\mathrm{S}_{8}$ & 27.5 & 25.7 & 101.6 & 1.05 & 0.28 & 0.28 & -91 & -15 \\
\hline
\end{tabular}


(a)

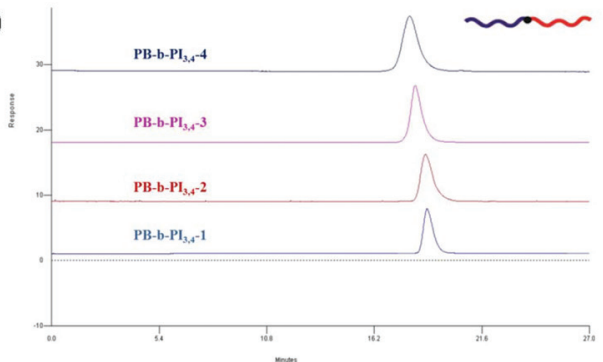

living blocks with the corresponding chlorosilane $\left(\mathrm{CH}_{3} \mathrm{SiCl}_{3}\right.$ or $\left.\mathrm{SiCl}_{4}\right)$. The molecular characterization via ${ }^{1} \mathrm{H}-\mathrm{NMR}$ spectroscopy was necessary to verify the composition results as revealed by SEC and MO, as well as to confirm the existence of the desirable 3,4-microstructure at high values $(>55 \%)$ for the PI segments and the 1,4-microstructure for the PB blocks $(\sim 92 \%)$ in all synthesized materials.

In Table S1, $\dagger$ the type and number of protons with the corresponding chemical shifts for protons incorporated in the monomeric units of polybutadiene and polyisoprene are presented. Three representative spectra corresponding to samples $\mathrm{PB}_{1,4}-b-\mathrm{PI}_{3,4}-4, \quad \mathrm{~PB}_{1,4}\left(\mathrm{PI}_{3,4}\right)_{2}-\mathrm{S} 7$, and $\mathrm{PB}_{1,4}\left(\mathrm{PI}_{3,4}\right)_{3}$-S8 (corresponding to set no. 4) are given in Fig. 3. For each sample, the corresponding ${ }^{1} \mathrm{H}$-NMR spectrum of initial block $\mathrm{PB}_{1,4}$, intermediate $\mathrm{PI}_{3,4}$, and the final linear or non-linear copolymer is given for comparison reasons. The mass fractions and characteristic microstructure content of each arm (PB and PI), as calculated directly from the ${ }^{1} \mathrm{H}-\mathrm{NMR}$ spectra, are shown in Table S2. $\dagger$ High 3,4-microstructure (57-62\%) was obtained for all the PI blocks of the twelve (12) synthesized materials, while all PB blocks were enriched in 1,4-microstructure (90-92\%).

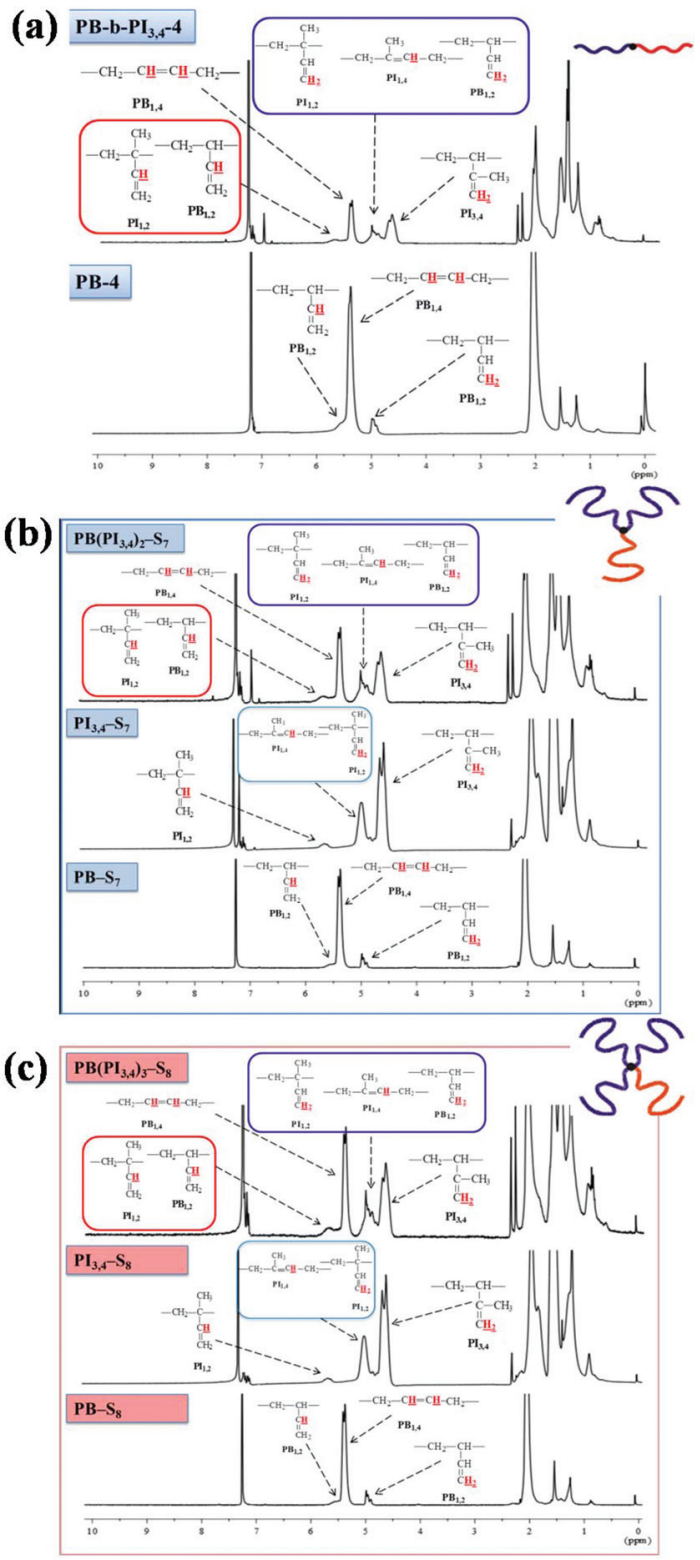

Fig. $3{ }^{1} \mathrm{H}-\mathrm{NMR}$ spectra for the three samples of set no. 4, corresponding to: (a) the $\mathrm{PB}_{1,4}-b-\mathrm{PI}_{3,4}-4$ diblock copolymer and the initial PB-4 homopolymer, (b) the $\mathrm{PB}_{1,4}\left(\mathrm{PI}_{3,4}\right)_{2}-\mathrm{S}_{7}$ miktoarm star copolymer and the respective $\mathrm{PB}-\mathrm{S}_{7}, \mathrm{Pl}_{3,4}-\mathrm{S}_{7}$ blocks and (c) the $\mathrm{PB}_{1,4}\left(\mathrm{PI}_{3,4}\right)_{3}-\mathrm{S}_{8}$ miktoarm star copolymer and the respective $\mathrm{PB}-\mathrm{S}_{8}, \mathrm{Pl}_{3,4}-\mathrm{S}_{8}$ blocks.

\section{Thermal characterization results of linear diblock and non- linear miktoarm star copolymers}

Analysing the DSC results of all samples, two endothermic transitions were obtained, corresponding to the $T_{\mathrm{g}}$ of $\mathrm{PB}_{1,4}$ and $\mathrm{PI}_{3,4}$. Crystallization and melting temperatures $\left(T_{\mathrm{m}}\right)$ are not evident in any thermograph, since both PB and PI, as synthesized by anionic polymerization with the specific microstructures, exhibit $T_{\mathrm{m}}$ above $40{ }^{\circ} \mathrm{C}$ and the non-linear microstructures $(-1,2$ and $-3,4)$ are mostly atactic with very low degree of crystallization. The existence of two $T_{\mathrm{g}}$, similar to the $T_{\mathrm{g}}$ of the corresponding homopolymers ${ }^{27}$ leads to the con- 


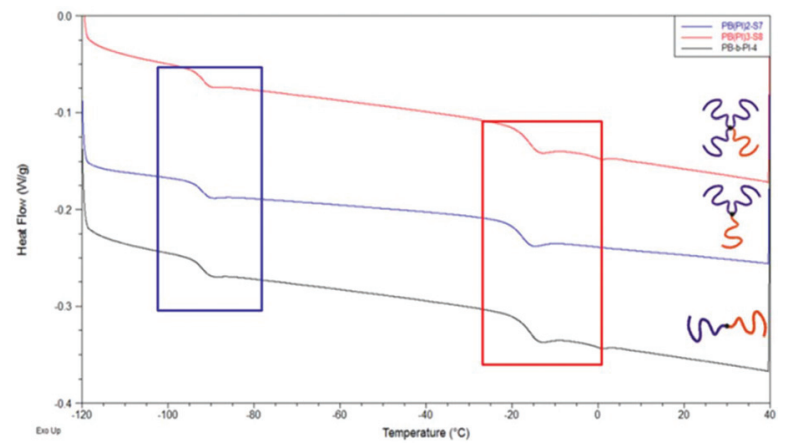

Fig. 4 DSC thermographs for samples constituting set no. 4, where $\mathrm{PB}_{1,4}-b-\mathrm{PI}_{3,4}-4, \mathrm{~PB}_{1,4}\left(\mathrm{PI}_{3,4}\right)_{2}-\mathrm{S}_{7}$ and $\mathrm{PB}_{1,4}\left(\mathrm{Pl}_{3,4}\right)_{3}-\mathrm{S}_{8}$ are the black, blue and red colour curves respectively. In the blue frame the glass transition temperatures for all the PB blocks are presented and in the red frame the glass transition temperatures of the PI block are depicted.

clusion that the two different blocks are totally immiscible. All $T_{\mathrm{g}}$ of the miktoarm star copolymers and the corresponding linear diblock copolymers, along with their molecular characteristics are also presented in Table 1. A typical DSC thermograph including the $\mathrm{PB}_{1,4}-b-\mathrm{PI}_{3,4}-4, \quad \mathrm{~PB}_{1,4}\left(\mathrm{PI}_{3,4}\right)_{2}-\mathrm{S}_{7}$ and $\mathrm{PB}_{1,4}\left(\mathrm{PI}_{3,4}\right)_{3}-\mathrm{S}_{8}$ relevant to set no. 4 is given in Fig. 4 . The three different DSC thermographs are presented in one plot for comparison reasons. The remaining DSC thermographs for the other three sets of samples (set no. 1, 2 and 3 respectively), are given in the ESI (Fig. S5-S7†).

\section{Morphological characterization results of linear diblock and non-linear miktoarm star copolymers}

The morphological characterization of all synthesized polymers was accomplished exclusively by TEM studies. It should be noted that this type of copolymers cannot be studied with small angle X-ray scattering (SAXS) due to the similar and almost identical electron density of the two polydiene segments involved. ${ }^{33,43}$

The sample preparation used to investigate the morphology of all final copolymers in bulk is a crucial and important procedure. The casting was performed in a non-selective solvent for several days to promote the formation of equilibrium morphologies. $^{22,44}$ Initially, for the preparation of the thin films, a $5 \% \mathrm{w} / \mathrm{v}$ solution of each sample in toluene was prepared. The samples were casted for approximately 5-7 days in a properly established saturated environment. Films with a thickness approximately $1 \mathrm{~mm}$ were formed and parts of them were placed in an oven for thermal annealing $\left(50{ }^{\circ} \mathrm{C}\right)$ for 5 days. Afterwards, each film was removed from the annealing oven and immersed in liquid nitrogen for a few seconds to keep the adopted structure at a high annealing temperature. The quenched films were directly cryo-ultramicrotomed, and thin films of approximately $40 \mathrm{~nm}$ were collected on copper grids. In order to enhance the intrinsic difference in electron density between the PB and PI blocks, selective staining with aqueous solution of $\mathrm{OsO}_{4}$ for approximately 1 hour, was employed. PI chains are stained less, since the double bonds of $\mathrm{PI}_{3,4}$ segments are sterically hindered compared to those of $\mathrm{PB}_{1,4}$ domains. ${ }^{22,27,33}$

Another important aspect of the $\mathrm{PB}_{1,4} / \mathrm{PI}_{3,4}$ copolymers system is that the interaction parameter $\chi$ is yet unknown. Furthermore, the restrictions concerning the electron densities of both PB and PI, do not allow SAXS measurements in order to calculate the interaction parameter $\chi$, by studying the orderdisorder transition as a function of temperature.

\section{Set no. 1}

The TEM images obtained from the stained sections of $\mathrm{PB}_{1,4}-b$ $\mathrm{PI}_{3,4}-1, \quad \mathrm{~PB}_{1,4}\left(\mathrm{PI}_{3,4}\right)_{2}-\mathrm{S}_{1}$ and $\mathrm{PB}_{1,4}\left(\mathrm{PI}_{3,4}\right)_{3}-\mathrm{S}_{2}$ copolymers after being thermally annealed are presented in Fig. 5. As far as the $\mathrm{PB}_{1,4}-b-\mathrm{PI}_{3,4}-1$ diblock copolymer is concerned, alternating lamellae of the two different phases were adopted as illustrated in the TEM image (Fig. 5a). Alternating dark grey and white/grey layers are evident in which the dark layer $\left(\mathrm{PB}_{1,4}\right)$ is slightly smaller in dimensions when compared with the other layer, PI, verifying therefore the volume fraction $\left(0.42\right.$ for $\left.\mathrm{PB}_{1,4}\right)$ as calculated from ${ }^{1} \mathrm{H}-\mathrm{NMR}$. Moreover, no discrepancy is encountered with the morphology predicted by the phase diagram of the PS- $b$-PI diblock copolymer system. ${ }^{45-47}$ In the TEM image (Fig. 5b) from thin sections of the annealed $\mathrm{PB}_{1,4}$ $\left(\mathrm{PI}_{3,4}\right)_{2} \mathrm{~S}_{1}$ sample with $\mathrm{PB}_{1,4}$ volume fraction equal to 0.41 , hexagonally close-packed cylinders of the dark grey phase $\left(\mathrm{PB}_{1,4}\right)$ in the white matrix $\left(\mathrm{PI}_{3,4}\right)$ are evident. The TEM image (Fig. 5c) from the annealed $\mathrm{PB}_{1,4}\left(\mathrm{PI}_{3,4}\right)_{3}-\mathrm{S}_{2}$ sample, with $\mathrm{PB}_{1,4}$ volume fraction equal to 0.40 , indicates bcc spheres of the dark grey $\mathrm{PB}_{1,4}$ domains in a white $\mathrm{PI}_{3,4}$ matrix.

The image does not show any alternating layers (as in the hcp cylinders), and since no SAXS experiments are possible, based on the phase diagram for non-linear copolymers of the

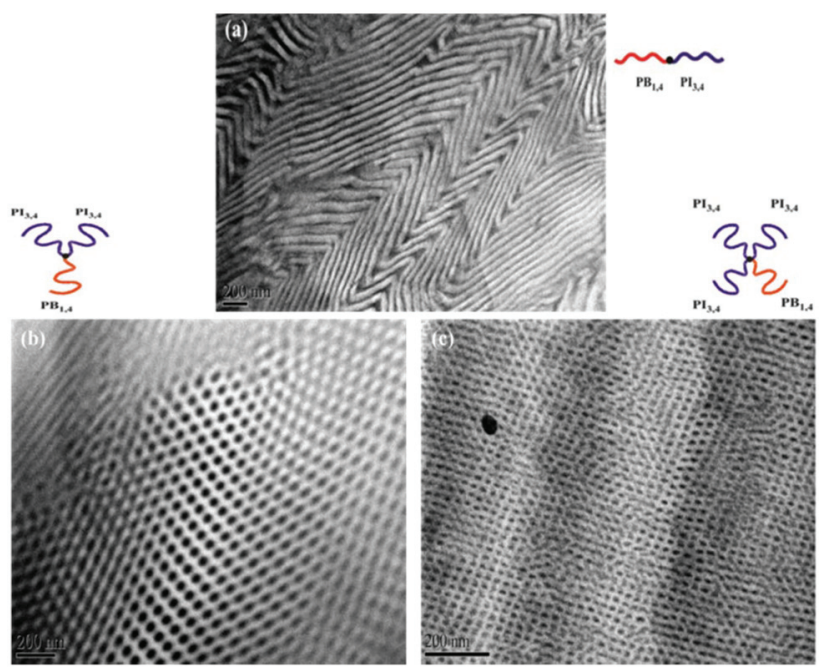

Fig. 5 Bright-field TEM images of the first set of the $\mathrm{PB}_{1,4}\left(\mathrm{PI}_{3,4}\right)_{n=1,2,3}$ type samples after thermal annealing at $50{ }^{\circ} \mathrm{C}$ for 5 days followed by microtoming and staining with vapors of $\mathrm{OsO}_{4}$ for approximately 1 hour corresponding to: (a) $\mathrm{PB}_{1,4}-b-\mathrm{PI}_{3,4}-1$ diblock copolymer, (b) $\mathrm{PB}_{1,4}\left(\mathrm{PI}_{3,4}\right)_{2^{-}}$ $\mathrm{S}_{1}$ miktoarm star copolymer and (c) $\mathrm{PB}_{1,4}\left(\mathrm{Pl}_{3,4}\right)_{3}-\mathrm{S}_{2}$ miktoarm star copolymer. 
PS/PI system, the TEM is significantly indicating bcc spheres of minority component (PB) in the matrix of the majority (PI).

Set no. 2

In Fig. 6 the TEM images obtained from the stained sections of samples $\mathrm{PB}_{1,4}-b-\mathrm{PI}_{3,4}-2, \mathrm{~PB}_{1,4}\left(\mathrm{PI}_{3,4}\right)_{2}-\mathrm{S}_{3}$ and $\mathrm{PB}_{1,4}\left(\mathrm{PI}_{3,4}\right)_{3}-\mathrm{S}_{4}$, after being thermally annealed are presented. In all cases, the $\mathrm{PB}_{1,4}$ domains are the majority component since the volume fractions (Table 1) for $\mathrm{PB}_{1,4}$ are $0.71,0.67$ and 0.69 , respectively. For the linear diblock copolymer $\mathrm{PB}_{1,4}-b-\mathrm{PI}_{3,4}-2$, hexagonally close-packed cylinders of the minority component (PI) in the matrix of the majority (PB) are evident (Fig. 6a). A cubic structure based on the $\mathrm{G}$ minimal surface (double gyroid) is expected at the SSL (strong segregation limit) for the PS- $b$-PI diblock copolymer for similar volume fraction (0.29 of the minority component) taking into consideration the relevant phase diagram $\chi N$ vs. $f(\varphi)$, where $\chi$ is the Flory-Huggins interaction parameter, $N$ the total degree of polymerization and $\varphi$ the volume fraction of the minority component. ${ }^{45-47}$ For both annealed miktoarm star copolymers $\mathrm{PB}_{1,4}\left(\mathrm{PI}_{3,4}\right)_{2}-\mathrm{S}_{3}$ and $\mathrm{PB}_{1,4}\left(\mathrm{PI}_{3,4}\right)_{3}-\mathrm{S}_{4}$ alternating lamellar structures were observed, where the dark grey area corresponds to the $\mathrm{PB}_{1,4}$ phase and the white to the $\mathrm{PI}_{3,4}$ domains (Fig. $6 \mathrm{~b}$ and c respectively).

\section{Set no. 3}

Further TEM studies were conducted and are presented in Fig. 7, for the third set of copolymers, specifically for the $\mathrm{PB}_{1,4^{-}}$ $b$ - $\mathrm{PI}_{3,4}-3 \quad \mathrm{~PB}_{1,4}\left(\mathrm{PI}_{3,4}\right)_{2}-\mathrm{S}_{5}$ and $\mathrm{PB}_{1,4}\left(\mathrm{PI}_{3,4}\right)_{3}-\mathrm{S}_{6}$ after thermal annealing at $50{ }^{\circ} \mathrm{C}$ for 5 days. Similar to the $\mathrm{PB}_{1,4}-b-\mathrm{PI}_{3,4}-1$, alternating lamellae of the two different phases was evident for sample $\mathrm{PB}_{1,4}-b-\mathrm{PI}_{3,4}-3$ (Fig. 7a). The only difference in the $\mathrm{PB}_{1,4}-b-\mathrm{PI}_{3,4}-3$ lies on the reversibility of the layer thickness where in this case the $\mathrm{PB}_{1,4}\left(\varphi_{\mathrm{PB}}=0.59\right)$ layer (dark grey) is

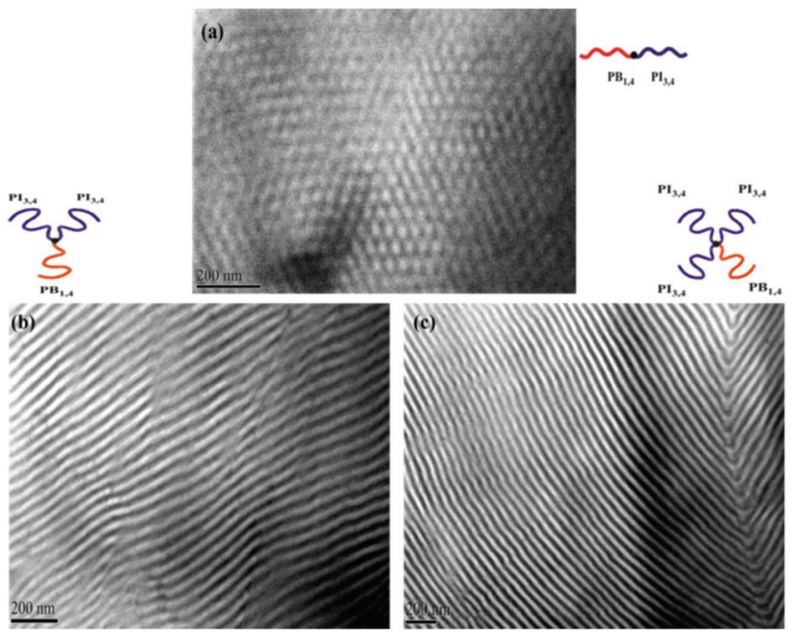

Fig. 6 Bright-field TEM images of the second set of the $\mathrm{PB}_{1,4}\left(\mathrm{PI}_{3,4}\right)_{n=1,2,3}$ type after thermal annealing at $50{ }^{\circ} \mathrm{C}$ for 5 days followed by microtoming and staining with vapors of $\mathrm{OsO}_{4}$ for approximately 1 hour corresponding to: (a) $\mathrm{PB}_{1,4}-b-\mathrm{PI}_{3,4}-2$ diblock copolymer, (b) $\mathrm{PB}_{1,4}\left(\mathrm{PI}_{3,4}\right)_{2}-\mathrm{S}_{3}$ miktoarm star copolymer and (c) $\mathrm{PB}_{1,4}\left(\mathrm{PI}_{3,4}\right)_{3}-\mathrm{S}_{4}$ miktoarm star copolymer.

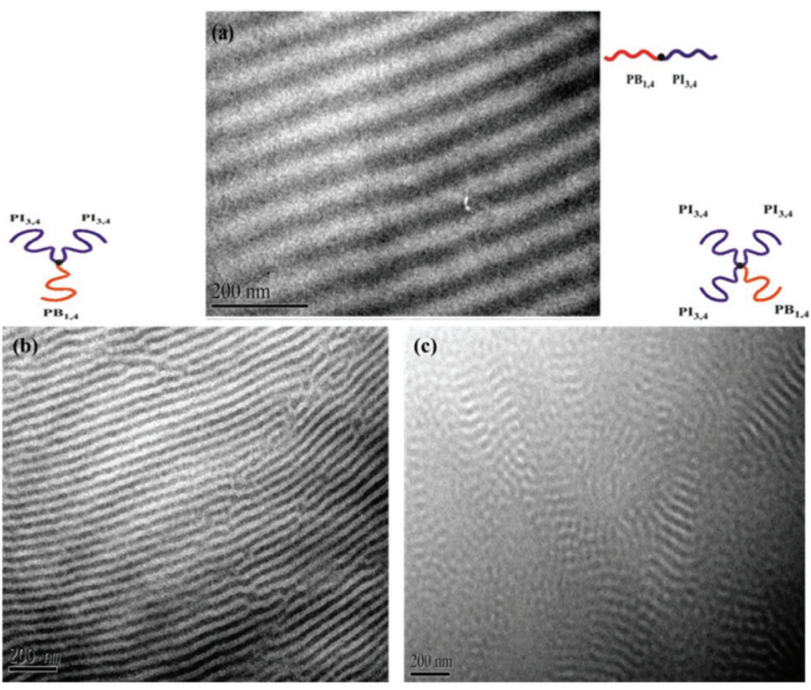

Fig. 7 Bright-field TEM images of the third set of the $\mathrm{PB}\left(\mathrm{PI}_{3,4}\right)_{n=1,2,3}$ type after thermal annealing at $50{ }^{\circ} \mathrm{C}$ for 5 days followed by microtoming and staining with vapors of $\mathrm{OsO}_{4}$ for approximately 1 hour corresponding to: (a) $\mathrm{PB}_{1,4}-b-\mathrm{Pl}_{3,4}-3$ diblock copolymer, (b) $\mathrm{PB}_{1,4}\left(\mathrm{PI}_{3,4}\right)_{2}-\mathrm{S}_{5}$ miktoarm star copolymer and (c) $\mathrm{PB}_{1,4}\left(\mathrm{PI}_{3,4}\right)_{3}-\mathrm{S}_{6}$ miktoarm star copolymer.

slightly larger than the PI layer, verifying, the volume fraction from the molecular characterization studies. No discrepancy is encountered with the adopted morphology for sample $\mathrm{PB}_{1,4}-b$ $\mathrm{PI}_{3,4}-3$ from that expected for a PS- $b$-PI diblock copolymer sample with identical molecular characteristics and composition. ${ }^{45-47}$ Similarly, TEM micrographs (Fig. 7b) from thin sections of the annealed $\mathrm{PB}_{1,4}\left(\mathrm{PI}_{3,4}\right)_{2}-\mathrm{S}_{5}$ sample with $\mathrm{PB}$ volume fraction equal to 0.57 showcase alternating lamellae of the dark grey phase $\left(\mathrm{PB}_{1,4}\right)$ and the white phase $\left(\mathrm{PI}_{3,4}\right)$. The TEM (Fig. 7c) image of the annealed $\mathrm{PB}_{1,4}\left(\mathrm{PI}_{3,4}\right)_{3}-\mathrm{S}_{6}$ sample with $\mathrm{PB}$ volume fraction equal to 0.56 illustrates hexagonally close-packed cylinders [dark grey phase $\left(\mathrm{PB}_{1,4}\right)$ in a white matrix $\left.\left(\mathrm{PI}_{3,4}\right)\right]$. For this sample, despite thermal annealing, no long-range order could be observed, as evident in the other related samples with different volume fractions. A possible explanation could be allocated to the fact that the thin sections were slightly mechanically deformed as taken from the microtome and were placed on the TEM grid. For all samples [except $\left.\mathrm{PB}_{1,4}\left(\mathrm{PI}_{3,4}\right)_{3}-\mathrm{S}_{6}\right]$ the TEM images are indicating distinct and straightforward microdomains with relevant long-range order.

\section{Set no. 4}

TEM results for samples $\mathrm{PB}_{1,4}-b-\mathrm{PI}_{3,4}-4, \mathrm{~PB}_{1,4}\left(\mathrm{PI}_{3,4}\right)_{2}-\mathrm{S}_{7}$ and $\mathrm{PB}_{1,4}\left(\mathrm{PI}_{3,4}\right)_{3}-\mathrm{S}_{8}$, after thermal annealing, are presented in Fig. 8. These materials compose the last set of samples with the lowest volume fraction of $\mathrm{PB}(0.32,0.30$ and 0.28 respectively). For the linear diblock $\mathrm{PB}_{1,4}-b-\mathrm{PI}_{3,4}-4$, dark grey hexagonally packed cylinders of $\mathrm{PB}_{1,4}$ in the white/grey matrix of the PI phase was the adopted morphology (Fig. 8a). Since the volume fraction of $\mathrm{PB}_{1,4}$ is equal to 0.32 , according to the morphological behaviour of the already studied PS- $b$-PI system, a cubic 
structure (double gyroid), with networks of the minority component (PB) in the matrix of the majority (PI) should be observed. ${ }^{45-47}$

Since $\mathrm{PB}_{1,4}$ and $\mathrm{PI}_{3,4}$ are very flexible chains, the adaptation of such a complex 3D morphology may not be possible due to entropic and enthalpic constrains, leading to a less thermodynamically demanding 2D topology. Therefore, hexagonally close-packed (hcp) cylinders of the minority phase (PB) in the matrix of the majority (PI) are preferred. In this case, it would be interesting to prepare binary blends of the diblock copolymers, with either homopolymer $\mathrm{PB}_{1,4}$ or homopolymer $\mathrm{PI}_{3,4}$ in order to explore whether the double gyroid morphology will appear in the volume fraction regime 0.25-0.32. If not, then the specific studied system indicates a major discrepancy with the microphase separation of the well-studied PS- $b-\mathrm{PI}^{42-45}$ diblock copolymer system.

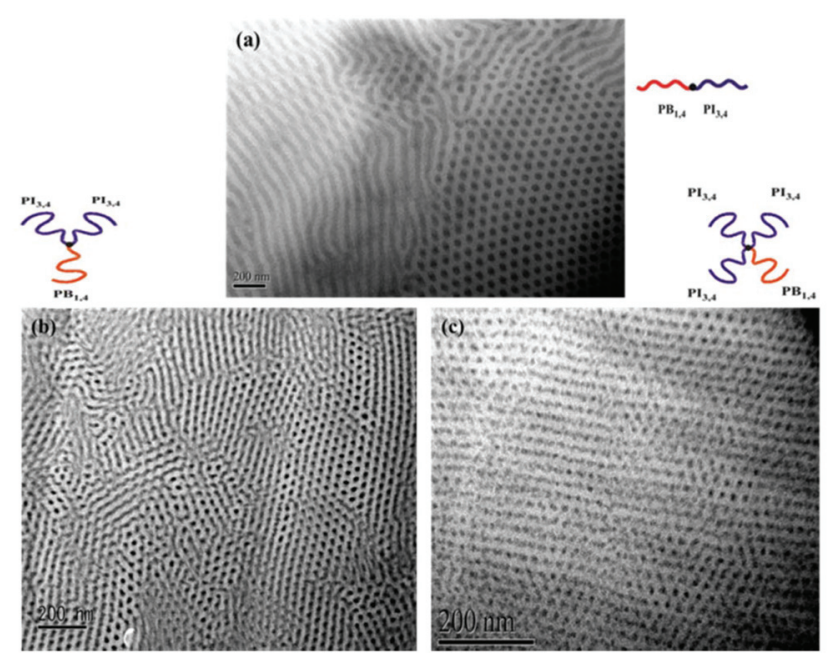

Fig. 8 Bright-field TEM images of the fourth set of the $\mathrm{PB}_{1,4}\left(\mathrm{Pl}_{3,4}\right)_{n=1,2,3}$ type after thermal annealing at $50{ }^{\circ} \mathrm{C}$ for 5 days followed by microtoming and staining with vapors of $\mathrm{OsO}_{4}$ for approximately 1 hour corresponding to: (a) $\mathrm{PB}_{1,4}-b-\mathrm{PI}_{3,4}-4$ diblock copolymer, (b) $\mathrm{PB}_{1,4}\left(\mathrm{PI}_{3,4}\right)_{2}-\mathrm{S}_{7}$ miktoarm star copolymer and (c) $\mathrm{PB}_{1,4}\left(\mathrm{Pl}_{3,4}\right)_{3}-\mathrm{S}_{8}$ miktoarm star copolymer.
For the $\mathrm{PB}_{1,4}\left(\mathrm{PI}_{3,4}\right)_{2}-\mathrm{S}_{7}$, the TEM image (Fig. 8b) clearly indicates hexagonally close-packed cylinders of the dark grey phase $\left(\mathrm{PB}_{1,4}\right)$ in the white matrix $\left(\mathrm{PI}_{3,4}\right)$, whereas the corresponding image of the annealed $\mathrm{PB}_{1,4}\left(\mathrm{PI}_{3,4}\right)_{3}-\mathrm{S}_{8}$ shows bcc spheres of the dark grey phase $\left(\mathrm{PB}_{1,4}, \varphi_{\mathrm{PB}}=0.28\right)$ in a white matrix $\left(\mathrm{PI}_{3,4}\right)$. The image does not show any alternating layers (as in the hep cylinders), and since no SAXS experiments are possible, we rely on the fact (by tilting) that the TEM micrograph in Fig. 8c indicates exclusively bcc spheres of the minority component (PB) in the matrix of the majority (PI).

In Table 2, the molecular characteristics, volume fractions, degree of polymerization $(N)$, theoretically predicted morphologies based on $\mathrm{PS}(\mathrm{PI})_{n}$ (where $n=1,2,3$ ) system, ${ }^{42,44,45}$ and verification of the adopted morphology for all the linear and non-linear polydiene copolymers $\mathrm{PB}(\mathrm{PI})_{n}$ (where $n=1,2$, 3), are summarized.

It is straightforward from Table 2 that for each set of samples, copolymers with identical molecular characteristics (almost constant total number average molecular weight) have been synthesized in order to be compared based on the complexity of the architecture, as well as on the differentiation in adopted topology for identical samples indicating different volume fractions. It is the first time that such a study is being reported directly for linear and non-linear materials composed exclusively of immiscible polydienes $\left(\mathrm{PB}_{1,4}\right.$ and $\left.\mathrm{PI}_{3,4}\right)$, which microphase separate.

The results between the theoretically predicted morpho$\log y^{17,47}$ and the experimentally observed by TEM, did not lead to any discrepancies for the novel miktoarm stars, when compared with the corresponding results for similar linear and non-linear PS/PI systems, as evident from the last column of Table 2. It was expected that such an agreement would occur only for the linear diblock copolymers, or even for the least complex architecture [samples of the $\mathrm{PB}_{1,4}\left(\mathrm{PI}_{3,4}\right)_{2}$ type]. The fact that $\mathrm{PB}_{1,4}\left(\mathrm{PI}_{3,4}\right)_{3}$ type of copolymers also showed similar agreement is of great importance, since it verifies our assumption regarding identical Kuhn lengths between the two polydienes, leading to elasticity parameters $(\varepsilon)$ exclusively dependant on the number of chains.

Table 2 Molecular characteristics, volume fractions, degrees of polymerization $(N)$, theoretically predicted morphologies and verification of morphology for all the copolymers synthesized (the symbol * indicates morphology of low long-range order)

\begin{tabular}{|c|c|c|c|c|c|c|c|}
\hline Sample sets & Samples & $\left(\bar{M}_{\mathrm{n}}\right)_{\text {total }}\left(\mathrm{kg} \mathrm{mol}{ }^{-1}\right)$ & $\varphi_{\mathrm{PB}}$ & $\varphi_{\mathrm{PI}}$ & $N_{\mathrm{PB} 1,4 / \mathrm{PI} 3.4}$ & Morphology from theory & Verified morphology \\
\hline \multirow[t]{3}{*}{ Set no. 1} & $\mathrm{~PB}_{1,4}-b-\mathrm{PI}_{3.4}-1$ & 93.6 & 0.42 & 0.58 & 1522 & LAM & $\checkmark$ \\
\hline & $\mathrm{PB}_{1,4}\left(\mathrm{PI}_{3.4}\right)_{2}-\mathrm{S}_{1}$ & 107.3 & 0.41 & 0.59 & 1836 & $\mathrm{CYL}_{\mathrm{PB}}$ & $\checkmark$ \\
\hline & $\mathrm{PB}_{1,4}\left(\mathrm{PI}_{3.4}\right)_{3}-\mathrm{S}_{2}$ & 109.4 & 0.40 & 0.60 & 1848 & $\mathrm{SPH}_{\mathrm{PB}}$ & $\checkmark$ \\
\hline \multirow[t]{3}{*}{ Set no. 2} & $\mathrm{~PB}_{1,4}-b-\mathrm{PI}_{3.4}-2$ & 92.4 & 0.71 & 0.29 & 1606 & DG & $\mathrm{CYL}_{\mathrm{PI}}$ \\
\hline & $\mathrm{PB}_{1,4}\left(\mathrm{PI}_{3.4}\right)_{2}-\mathrm{S}_{3}$ & 92.4 & 0.67 & 0.33 & 1631 & LAM & $\checkmark$ \\
\hline & $\mathrm{PB}_{1,4}\left(\mathrm{PI}_{3.4}\right)_{3}-\mathrm{S}_{4}$ & 88.2 & 0.69 & 0.31 & 1571 & LAM & $\checkmark$ \\
\hline \multirow[t]{3}{*}{ Set no. 3} & $\mathrm{~PB}_{1,4}-b-\mathrm{PI}_{3.4}-3$ & 98.5 & 0.59 & 0.41 & 1670 & LAM & $\checkmark$ \\
\hline & $\mathrm{PB}_{1,4}\left(\mathrm{PI}_{3.4}\right)_{2}-\mathrm{S}_{5}$ & 98.1 & 0.57 & 0.43 & 1692 & LAM & $\checkmark$ \\
\hline & $\mathrm{PB}_{1,4}\left(\mathrm{PI}_{3.4}\right)_{3}-\mathrm{S}_{6}$ & 100.3 & 0.56 & 0.44 & 1709 & $\mathrm{CYL}_{\mathrm{PB}}$ & $\sqrt{ }^{*}$ \\
\hline \multirow[t]{3}{*}{ Set no. 4} & $\mathrm{~PB}_{1,4}-b-\mathrm{PI}_{3.4}-4$ & 107.9 & 0.32 & 0.68 & 1722 & DG & $\mathrm{CYL}_{\mathrm{PB}}$ \\
\hline & $\mathrm{PB}_{1,4}\left(\mathrm{PI}_{3.4}\right)_{2}-\mathrm{S}_{7}$ & 95.5 & 0.30 & 0.70 & 1523 & $\mathrm{CYL}_{\mathrm{PB}}$ & $\checkmark$ \\
\hline & $\mathrm{PB}_{1,4}\left(\mathrm{PI}_{3.4}\right)_{3}-\mathrm{S}_{8}$ & 101.6 & 0.28 & 0.72 & 1643 & $\mathrm{SPH}_{\mathrm{PB}}$ & $\checkmark$ \\
\hline
\end{tabular}

LAM, alternating lamellae; CYL, hexagonally packed cylinders; SPH, spheres; DG, double gyroid. 
The only cases where different results were obtained from the relevant PS/PI system, was for two linear copolymers where in both samples, the expected morphology should have been the DG cubic structure (sample-2 and sample-4). In contrast, hexagonally close-packed cylinders of the minority component ( $\mathrm{PB}$ or $\mathrm{PI}$ for $\varphi_{\mathrm{PB}}=0.71$ and 0.32 respectively) in the matrix (PI or $\mathrm{PB}$ ) were observed.

As already described for the specific diblock copolymers, since $\mathrm{PB}_{1,4}$ and $\mathrm{PI}_{3,4}$ exhibit high chain flexibility, their ability to adopt such a complex architecture (DG) may not be possible due to entropic as well as enthalpic constrains. As a result, this leads to a less thermodynamically demanding topology such as the hcp cylinders of the minority phase in the matrix of the majority.

Moreover, rheological studies of the aforementioned synthesized materials would be of great scientific interest, since elastomers are materials which are capable of undergoing extremes on stress/strain mechanical properties studies and will provide further insight into polymer dynamics. We aim, in order to complete the structure property relationship of the specific copolymer system, to calculate the $\chi_{\text {eff }}$ with SANS and rheology experiments as well as conclude to a more detailed phase diagram by including the morphologies adopted from the binary blends of the pure linear and non-linear copolymers with corresponding homopolymers of $\mathrm{hPB}_{1,4}$ or $\mathrm{hPI}_{3,4}$ respectively.

\section{Conclusions}

A series of model linear and non-linear copolymers of the $\mathrm{PB}_{1,4}\left(\mathrm{PI}_{3,4}\right)_{n}$ type (where $n=1,2,3$ ) was successfully synthesized by employing anionic polymerization high-vacuum techniques in combination with selective chlorosilane chemistry. Specifically, twelve (12) samples were prepared and divided into four (4) different sets, where each set includes one linear diblock copolymer with similar molecular characteristics to the corresponding $\mathrm{PB}_{1,4}\left(\mathrm{PI}_{3,4}\right)_{2}$ and $\mathrm{PB}_{1,4}\left(\mathrm{PI}_{3,4}\right)_{3}$ miktoarm stars. Molecular characterization was performed through SEC, MO, and ${ }^{1} \mathrm{H}-\mathrm{NMR}$ measurements, indicating a high degree of molecular and compositional homogeneity in all cases. DSC and TEM studies verified the microphase separation and provide significant information concerning the influence of the architecture (linear or non-linear) on the adopted topology. Morphological characterization studies also revealed the coherence of theoretical [for the $\mathrm{PS}(\mathrm{PI})_{n}$ system] and experimental [for the $\mathrm{PB}_{1,4}\left(\mathrm{PI}_{3,4}\right)_{n}$ system] results for the complex architectures. The only discrepancies from the relevant PS/PI system were found for two linear copolymers, where in both samples, hcp cylinders of the minority phase in the matrix of the majority were observed, instead of the expected DG cubic structure morphology.

\section{Author contributions}

The manuscript was written through contributions of all authors. All authors have given approval to the final version of the manuscript.

\section{Conflicts of interest}

There are no conflicts to declare.

\section{Acknowledgements}

A. F. N. and A. A. acknowledge financial support from the Ministry of Science and Higher Education of the Russian Federation within State Contract 075-15-2019-1889. K. N., D. M., G. Z., I. M., K. T., G.-M. M. and A. A. would like to acknowledge the Network of Research Supporting Laboratories at the University of Ioannina for using the Electron Microscopy Facility and the Nuclear Magnetic Resonance Spectroscopy Center. K. N., G. Z. and N. H. would like to acknowledge the support of King Abdullah University of Science and Technology (KAUST).

\section{Notes and references}

1 J. H. Lee, C. Y. Koh, J. P. Singer, S. J. Jeon, M. Maldovan, O. Stein and E. L. Thomas, Adv. Mater., 2014, 26, 532-569.

2 I. W. Hamley, Angew. Chemie, 2003, 115, 1730-1752.

3 H. Hu, M. Gopinadhan and C. O. Osuji, Soft Matter, 2014, 10, 3867-3889.

4 P. A. Mistark, S. Park, S. E. Yalcin, D. H. Lee, O. Yavuzcetin, M. T. Tuominen, T. P. Russell and M. Achermann, ACS Nano, 2009, 3, 3987-3992.

5 L. Leibler, Macromolecules, 1980, 13, 1602-1617.

6 C. Park, J. Yoon and E. L. Thomas, Polymer, 2003, 44, 67256760.

7 T. Y. Lo, M. R. Krishnan, K. Y. Lu and R. M. Ho, Prog. Polym. Sci., 2018, 77, 19-68.

8 R. A. Segalman, B. McCulloch, S. Kirmayer and J. J. Urban, Macromolecules, 2009, 42, 9205-9216.

9 G. Polymeropoulos, G. Zapsas, K. Ntetsikas, P. Bilalis, Y. Gnanou and N. Hadjichristidis, Macromolecules, 2017, 50, 1253-1290.

10 S. Ito, R. Goseki, T. Ishizone and A. Hirao, Eur. Polym. J., 2013, 49, 2545-2566.

11 K. Matyjaszewski, Macromolecules, 2012, 45, 4015-4039.

12 M. W. Bates, S. M. Barbon, A. E. Levi, R. M. Lewis, H. K. Beech, K. M. Vonk, C. Zhang, G. H. Fredrickson, C. J. Hawker and C. M. Bates, ACS Macro Lett., 2020, 9, 396403.

13 A. E. Levi, L. Fu, J. Lequieu, J. D. Horne, J. Blankenship, S. Mukherjee, T. Zhang, G. H. Fredrickson, W. R. Gutekunst and C. M. Bates, Macromolecules, 2020, 53, 702-710.

14 J. Park, S. Jang and J. K. Kim, J. Polym. Sci., Part B: Polym. Phys., 2015, 53, 1-21.

15 N. Hadjichristidis, H. Iatrou, S. K. Behal, J. J. Chludzinski, M. M. Disko, R. T. Garner, K. S. Liang, D. J. Lohse and S. T. Milner, Macromolecules, 1993, 26, 5812-5815.

16 F. L. Beyer, S. P. Gido, G. Velis, N. Hadjichristidis and N. B. Tan, Macromolecules, 1999, 32, 6604-6607. 
17 M. O. de la Cruz and I. C. Sanchez, Macromolecules, 1986, 19, 2501-2508.

18 A. M. Mayes and M. O. de la Cruz, Macromolecules, 1991, 24, 3975-3976.

19 S. T. Milner, Macromolecules, 1994, 27, 2333-2335.

20 M. D. Gehlsen, K. Almdal and F. S. Bates, Macromolecules, 1992, 25, 939-943.

21 J. Allgaier, R. N. Young, V. Efstratiadis and N. Hadjichristidis, Macromolecules, 1996, 29, 1794-1797.

22 G. Zapsas, D. Moschovas, K. Ntetsikas, S. Rangou, J. H. Lee, E. L. Thomas, N. E. Zafeiropoulos and A. Avgeropoulos, J. Polym. Sci., Part B: Polym. Phys., 2015, 53, 1238-1246.

23 R. E. Cohen and A. R. Ramos, Macromolecules, 1979, 12, 131-134.

24 R. E. Cohen and D. E. Wilfong, Macromolecules, 1982, 15, 370-375.

25 C. Neumann, V. Abetz and R. Stadler, Colloid Polym. Sci., 1998, 276, 19-27.

26 C. Neumann, D. R. Loveday, V. Abetz and R. Stadler, Macromolecules, 1998, 31, 2493-2500.

27 A. Avgeropoulos, S. Paraskeva, N. Hadjichristidis and E. L. Thomas, Macromolecules, 2002, 35, 4030-4035.

28 Y. Mogi, H. Kotsuji, Y. Kaneko, K. Mori, Y. Matsushita and I. Ichiro, Macromolecules, 1992, 25, 5408-5411.

29 S. P. Gido, D. W. Schwark, E. L. Thomas and M. do Carmo Gonçalves, Macromolecules, 1993, 26, 2636-2640.

30 A. Avgeropoulos, S. Rangou, V. Krikorian and E. L. Thomas, Macromol. Symp., 2008, 267, 16-20.

31 S. Rangou and A. Avgeropoulos, J. Polym. Sci., Part A: Polym. Chem., 2009, 47, 1567-1574.

32 S. Rangou, D. Moschovas, I. Moutsios, G.-M. Manesi, K. Tsitoni, P. V. Bovsunovskaya, D. A. Ivanov, E. L. Thomas and A. Avgeropoulos, Molecules, 2020, 25, 6030.
33 D. Moschovas, G. M. Manesi, A. Karydis-Messinis, G. Zapsas, K. Ntetsikas, N. E. Zafeiropoulos, A. A. Piryazev, E. L. Thomas, N. Hadjichristidis, D. A. Ivanov and A. Avgeropoulos, Nanomaterials, 2020, 10, 1497.

34 Y. Zhang and U. Wiesner, Macromol. Chem. Phys., 1998, 199, 1771-1784.

35 M. Langela, U. Wiesner, H. W. Spiess and M. Wilhelm, Macromolecules, 2002, 35, 3198-3204.

36 C. Oelschlaeger, J. S. Gutmann, M. Wolkenhauer, H. W. Spiess, K. Knoll and M. Wilhelm, Macromol. Chem. Phys., 2007, 208, 1719-1729.

37 O. Vernáez, S. Dagreou, B. Grassl and A. J. Müller, J. Polym. Sci., Part B: Polym. Phys., 2016, 54, 433-444.

38 V. Hirschberg, L. Faust, D. Rodrigue and M. Wilhelm, Macromolecules, 2020, 53, 5572-5587.

39 G. Liu, H. Sun, S. Rangou, K. Ntetsikas, A. Avgeropoulos and S.-Q. Wang, J. Rheol., 2013, 57, 89-104.

40 H. Sun, G. Liu, K. Ntetsikas, A. Avgeropoulos and S. Q. Wang, Macromolecules, 2014, 47, 5839-5850.

41 N. Hadjichristidis, H. Iatrou, S. Pispas and M. Pitsikalis, J. Polym. Sci., Part A: Polym. Chem., 2000, 38, 32113234.

42 D. Uhrig and J. W. Mays, J. Polym. Sci., Part A: Polym. Chem., 2005, 43, 6179-6222.

43 S. Jangareddy and R. A. Register, Macromolecules, 2020, 53, 9142-9151.

44 N. Hadjichristidis, H. Iatrou, M. Pitsikalis, S. Pispas and A. Avgeropoulos, Prog. Polym. Sci., 2005, 30, 725782.

45 M. W. Matsen and F. S. Bates, Macromolecules, 1996, 29, 1091-1098.

46 F. S. Bates and G. H. Fredrickson, Phys. Today, 1999, 52, 32-38.

47 M. W. Matsen, Macromolecules, 2012, 45, 2161-2165. 